\title{
Broad-band difference interferometer as a temperature sensor
}

\author{
Kazimierz Gut, ${ }^{* 1}$ Zbigniew Opilski ${ }^{1}$ \\ ${ }^{1}$ Department of Optoelectronics, Silesian University of Technology, Krzywoustego 2, 44-100 Gliwice, Poland
}

Received November 21, 2014; accepted November 28, 2014; published December 31, 2014

\begin{abstract}
The paper presents the characteristics of a broad-band difference interferometer (spectropolarimetric). When a waveguide layer introduces light from a spectral range, at the output structure there is a spectral distribution which depends on the parameters of the propagation wavelength. It shows the effect of temperature on the parameters describing the system.
\end{abstract}

In recent years chemical and biochemical sensors have been developed [1]. One important parameter of this type of measurements is temperature. The paper presents some concepts of using a single-mode polymer layer SU8 to monitor the temperature of a waveguide structure. In a typical differential interferometer, orthogonal mode fundamental TE and TM are analyzed for one wavelength propagating at different phase velocities [2]. The increasing availability of fiber optic spectrometers and broadband light sources with a high power allows to enter planar waveguides into a whole range of visible spectrum [3]. In the analyzed system an orthogonal mode propagates for wavelengths from $450 \mathrm{~nm}$ to $600 \mathrm{~nm}$. This type of arrangement is shown in [3-5] and called a "frequency-resolved", "broad-band" or "wavelength interrogation" Mach-Zehnder interferometer. Concerning the differential interferometer analogous system is considered. In the case of $\mathrm{TE}_{0}$ and $\mathrm{TM}_{0}$ modes interference and for the same optical power density $\mathrm{I}_{0}$ transmitted in both modes (if the plane of polarization of the output polarizer is set at an angle to the surface of the optical waveguide equal to $45^{\circ}$ ), the signal recorded by the detector $I(\lambda)$ can be expressed by the formula [6]:

$$
\mathrm{I}(\lambda)=\mathrm{I}_{\mathrm{o}}\{1+\cos [\Delta \phi(\lambda)]\}
$$

where $\Delta \phi(\mathrm{t})$ is the phase difference between the modes at the output of the waveguide for the wavelength $\lambda$.

In the course of propagation the difference of the phases between the modes $\Delta \phi(\lambda)$ is attained, which is a function of the length of the path of propagation $L$, difference of the effective refractive index $\left(\mathrm{N}(\lambda)_{\mathrm{TM}}-\mathrm{N}(\lambda)_{\mathrm{TE}}\right)$ and the wavelength $\lambda$ :

$$
\Delta \phi(\lambda)=(2 \pi / \lambda)\left(\mathrm{N}(\lambda)_{\mathrm{TE}}-\mathrm{N}(\lambda)_{\mathrm{TM}}\right)
$$

In order to satisfy the conditions of coherence of the light beam before entering the structure of the polarizer, it must be placed at an angle of $45^{\circ}$ to the perpendicular direction

*E-mail: kazimierz.gut @ polsl.pl of the waveguide surface. The second polarizer, placed before the spectrometer at the same angle $\left(45^{\circ}\right)$, provides light from both orthogonal modes to one plane of polarization, allowing to record the signal of interference (Fig. 1).

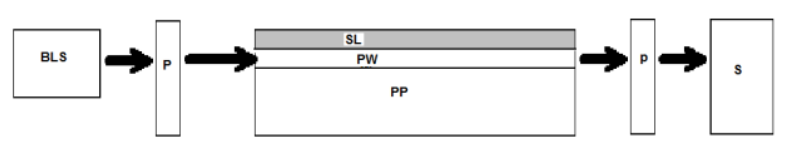

Fig. 1. Diagram of a broad-band interferometer: BLS - broadband light source, $\mathrm{PW}$ - planar waveguide, $\mathrm{PP}$ - substrate plate, $\mathrm{P}$ - polarizer, $\mathrm{S}$ spectrometer, SL - cover.

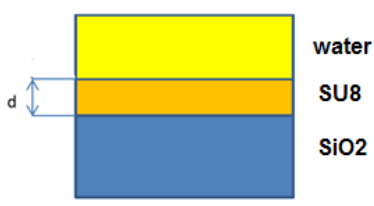

Fig. 2. Analyzed planar three-layer system.

In the numerical analysis a three-layer system has been used with the substrate $\left(\mathrm{SiO}_{2}\right)$, the waveguide layer (SU8), the cover (water) (Fig. 2).

The analysis takes into account the dispersion of a substrate and a waveguide layer (Fig. 3), which was determined by an ellipsometer SE850 SENTECH. The paper also deals with dispersion coating (water) [7].

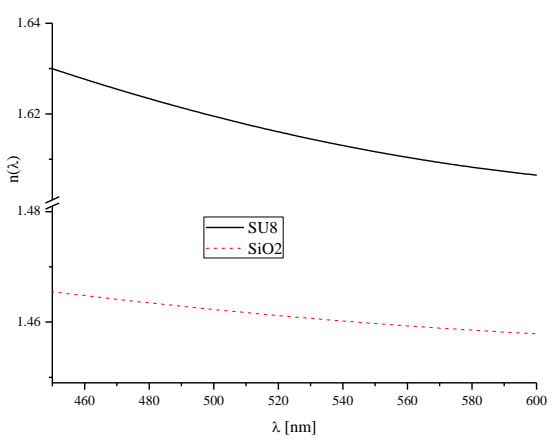

Fig. 3. Spectral characteristics of the refractive indices.

In the considered spectral range, refractive indices decrease monotonically, depressed with an increase in the length of the wave. 


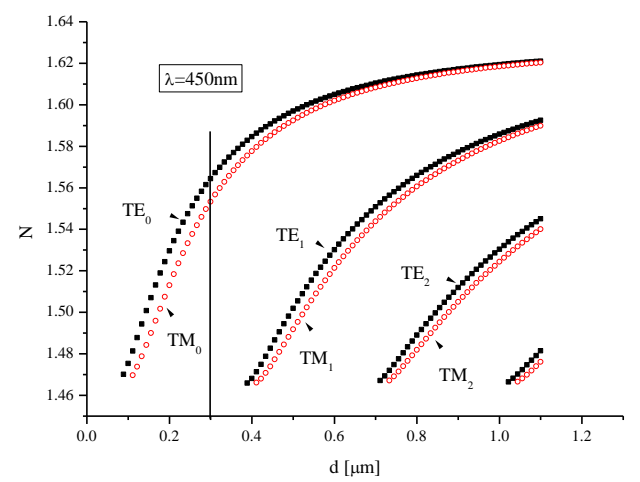

Fig. 4. Effective refractive indices as a function of the thickness $d$ of the layer SU8 at a wavelength of $\lambda=450 \mathrm{~nm}$.

In order to get pure propagation of the fundamental modes TE and TM in the structure, the effective refractive indices of the modes were determined as a function of thickness $d$ of the waveguide layer concerning the shortest wave length $\lambda=450 \mathrm{~nm}$ (Fig. 4 ).

For the waveguide layer of thickness $d=0.3 \mu \mathrm{m}$, it is possible to propagate only fundamental modes $\mathrm{TE}_{0}$ and $\mathrm{TM}_{0}$ (high order modes for the waveguide layer thickness cannot be propagated). This is also the case concerning longer waves in the entire range of the spectrum - from $0.45 \mu \mathrm{m}$ to $0.6 \mu \mathrm{m}$.

Using the software OPTIWAVE, effective refractive indices can be determined for the fundamental modes $\mathrm{TE}_{0}$ and $\mathrm{TM}_{0}$ in the considered wavelength range. Based on Eqs. (1)-(2), the spectral distribution of light intensity at the output of the system can be determined ( $\mathrm{I}_{0}$ assumed equal to 1 in the whole wavelength range).

Figure 5 presents the intensity of light distribution, concerning the propagation paths $0.5 \mathrm{~mm}, 1.0 \mathrm{~mm}$ and $2.0 \mathrm{~mm}$ long.

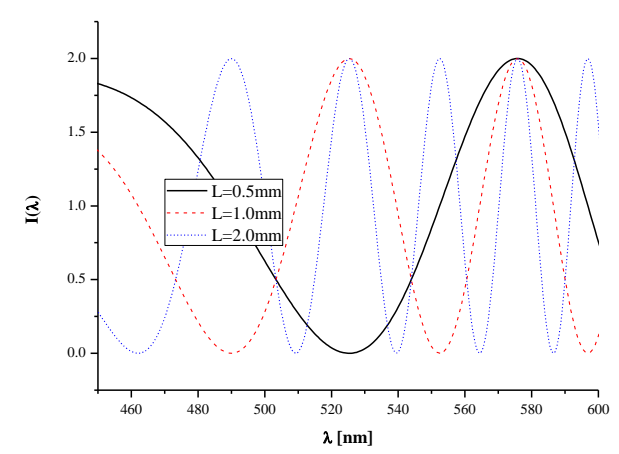

Fig. 5. Light intensity distribution $\mathrm{I}(\lambda)$ at the propagation distance of $0.5 \mathrm{~mm}, 1 \mathrm{~mm}$ and $2 \mathrm{~mm}$.
In the case of an interferential system, the most important parameter is the mode sensitivity $S\{n\}$, determining the changes of the effective refractive index $\Delta \mathrm{N}$ due to the changes of the refractive index of the cover $\Delta \mathrm{n}_{\mathrm{C}}$, substrate $\Delta \mathrm{n}_{\mathrm{S}}$, and the waveguide layer $\Delta \mathrm{n}_{\mathrm{F}}$ :

$$
S\left\{n_{C}\right\} \cong \frac{\Delta N}{\Delta n_{C}}, S\left\{n_{F}\right\} \cong \frac{\Delta N}{\Delta n_{C}}, S\left\{n_{S}\right\} \cong \frac{\Delta N}{\Delta n_{C}} .
$$

Changes in the effective refractive index can be connected with the changes in the refractive indices of the substrate, the cover and the waveguide layers [8-10]:

$$
\Delta N=\left(\frac{\partial N}{\partial n_{C}}\right) \Delta n_{C}+\left(\frac{\partial N}{\partial n_{S}}\right) \Delta n_{S}+\left(\frac{\partial N}{\partial n_{F}}\right) \Delta n_{F} \cdot
$$

Dividing the expression (4) by $\Delta \mathrm{T}$ we get [11]:

$$
\frac{\Delta N}{\Delta T}=\left(\frac{\partial N}{\partial n_{C}}\right) \frac{\Delta n_{C}}{\Delta T}+\left(\frac{\partial N}{\partial n_{S}}\right) \frac{\Delta n_{S}}{\Delta T}+\left(\frac{\partial N}{\partial n_{F}}\right) \frac{\Delta n_{F}}{\Delta T},
$$

where $\Delta n_{C} / \Delta T, \Delta n_{S} / \Delta T, \Delta n_{F} / \Delta T$ represent thermooptic (TO) coefficients of the substrate, cover and waveguide layer, respectively.

As follows from the paper [7], the TO coefficient for water in the considered wavelength range is equal to $-0.97 \cdot 10^{-4} 1 / \mathrm{K}$ and for a substrate $-1.28 \cdot 10^{-5} 1 / \mathrm{K}[11]$. The TO coefficient for the waveguide layer is determined from the ellipsometric measurements (Fig. 6).

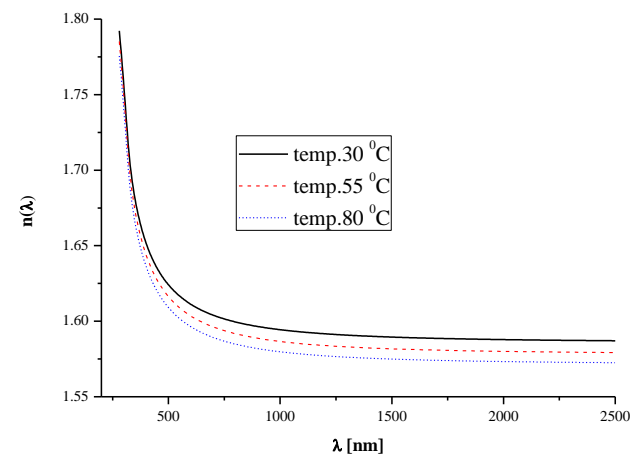

Fig 6. Dispersion of the refractive index of the waveguiding layer SU8 at different temperatures.

Figure 7 shows the difference of the refractive index of the SU8 layer at temperature changes from $30^{\circ} \mathrm{C}$ to $55^{\circ} \mathrm{C}$, from $55^{\circ} \mathrm{C}$ to $80^{\circ} \mathrm{C}$ and from $30^{\circ} \mathrm{C}$ to $80^{\circ} \mathrm{C}$ (ellipsometer SE850 SENTECH). 


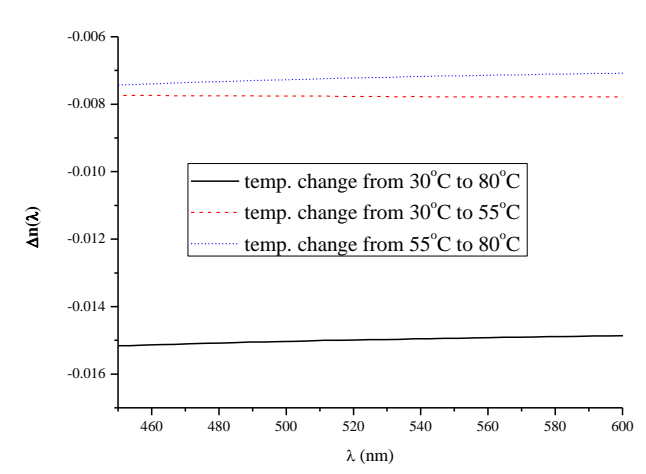

Fig 7. The difference in refraction at different temperature changes.

In this wavelength range the changes in the refractive index for the temperature range from $30^{\circ} \mathrm{C}$ to $55^{\circ} \mathrm{C}$ are almost constant and its average value is $\Delta \mathrm{n}=-0.00777$. Dividing the change in the refractive index by varying temperature, we obtain TO coefficient $\Delta \mathrm{n}_{\mathrm{F}} / \Delta \mathrm{T}=-3.1 \cdot 10^{-4}$ $1 / \mathrm{K}$. Please note that the TO coefficients waveguide layer and cover are negative, and the TO coefficient of the substrate is positive. The optical sensitivities $\mathrm{S}\left\{\mathrm{n}_{\mathrm{C}}\right\}$, $\mathrm{S}\left\{\mathrm{n}_{\mathrm{F}}\right\}, \mathrm{S}\left\{\mathrm{n}_{\mathrm{S}}\right\}$ are based on the dependence shown in [9]. Equation (5) determines the full thermal sensitivities $\mathrm{dN} / \mathrm{dT}$ of the fundamental TE and TM modes.

Effective refractive indices for the temperature $\mathrm{T}+\Delta \mathrm{T}$ can be determined from the equation:

$$
\mathrm{N}(\mathrm{T}+\Delta \mathrm{T})=\mathrm{N}(\mathrm{T})+\mathrm{dN} / \mathrm{dT} \times \Delta \mathrm{T}
$$

Figure 8 presents the light intensity distribution for the propagation length of $L=1 \mathrm{~mm}$ at temperatures $\mathrm{t}_{0}, \mathrm{t}_{0}+2^{\circ} \mathrm{C}$, $\mathrm{t}_{0}+4^{\circ} \mathrm{C}, \mathrm{t}_{0}+6^{\circ} \mathrm{C}$.

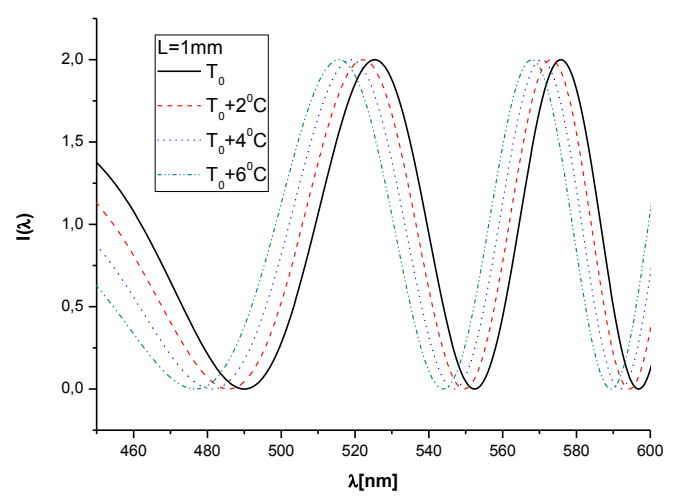

Fig. 8. Light intensity distribution $I_{n}(\lambda)$ for different temperatures.
In the broad-band (spectropolarimetric) interferometer the information about the value of temperature is connected with the spectral distribution transmitted by the system. A change of this parameter results in a monotonic shift of extremes values concerning the spectral distribution of light recorded by the spectrometer.

This work was supported by the Polish National Science Centre (NCN) under the grant 2012/07/B/ST7/01471.

\section{References}

[1] P. Kozma et al. Biosens. Bioelectron. 58, 287 (2014).

[2] K. Gut, Opt. Appl. 42, 407 (2012).

[3] K. Misakos et al. Opt. Expr. 22, 8856 (2014).

[4] M. Kitasara et al. Opt. Expr. 18, 8193 (2010).

[5] M. La Notte, V. Passaro, Sensor Actuat. B-Chem. 176, 994 (2013).

[6] K. Gut, Proc. SPIE 9291, 92910N (2014).

[7] M. Daimon, A. Masumura, Appl. Optics 46, 3811 (2007).

[8] K. Gut, S. Drewniak, Acta Physica Pol. A. 118, 1133 (2010).

[9] K. Tiefenthaler, W. Lukosz, J. Opt. Soc. Am. B 6, 209 (1989).

[10] K. Gut, S. Drewniak, Acta Physica Pol. A 120, 630 (2011).

[11] C. Delezoide et al. J. Opt. Soc. Am. B 31, 851 (2014). 